Annales Geophysicae (2002) 20: 185-190 (c) European Geophysical Society 2002

\title{
Faraday polarization fluctuations and their dependence on post sunset secondary maximum and amplitude scintillations at Delhi
}

\author{
J. K. Gupta, Lakha Singh, and R. S. Dabas \\ Radio and Atmospheric Sciences Division, National Physical Laboratory, Dr K. S. Krishnan Marg, New Delhi 110012, India
}

Received: 5 October 2000 - Revised: 21 June 2001 - Accepted: 3 September 2001

\begin{abstract}
VHF Faraday rotation (FR) and amplitude scintillation data recorded simultaneously during May 1978 December 1980 at Delhi $\left(28.63^{\circ} \mathrm{N}, 77.22^{\circ}\right.$ E; Dip $\left.42.44^{\circ} \mathrm{N}\right)$ is analyzed in order to study the Faraday polarization fluctuations (FPFs) and their dependence on the occurrence of post sunset secondary maximum (PSSM) and amplitude scintillations. It is noted that FPFs are observed only when both PSSM and scintillations also occur simultaneously. FPFs are observed only during winter and the equinoctial months of high sunspot years. FPFs events are associated with intense scintillation activity, which is characterized by sudden onsets and abrupt endings, and are observed one to three hours after the local sunset. When FPFs and scintillation data from Delhi is compared with the corresponding data from a still lower latitude station, Hyderabad $\left(17.35^{\circ} \mathrm{N}, 78.45^{\circ} \mathrm{E}\right)$, it is found that the occurrence of FPFs and scintillations at Delhi is conditional to their prior occurrence at Hyderabad, which indicates their production by a plasma bubble and the associated irregularities generated initially over the magnetic equator. In addition, FPFs and scintillation data for October 1979, when their occurrence was maximum, is also examined in relation to daytime (11:00 LT) electrojet strength $(\mathrm{EEj})$ values and evening hour h'F from an equatorial location, Kodaikanal $\left(10.3^{\circ} \mathrm{N}, 77.5^{\circ} \mathrm{E}\right)$. It is interesting to note that FPFs and scintillations are most likely observed when the EEj was $100 \mathrm{nT}$ or more and h'F reaches around $500 \mathrm{~km}$. These results show that $\mathrm{EEj}$ and evening hours h'F values over the magnetic equator are important parameters for predicting FPFs and scintillation activity at locations such as Delhi, where scintillation activity is much more intense as compared to the equatorial region due to the enhanced background ionization due to the occurrence of PSSM.
\end{abstract}

Key words. Ionosphere (equatorial ionosphere; ionospheric irregularities) - Radio science (ionospheric physics)

\section{Introduction}

The total electron content of the ionosphere (IEC) undergoes diurnal, seasonal and solar cycle variations. However,

Correspondence to: L. Singh (laksingh@ @ csnpl.ren.nic.in) in addition to the daytime maximum around 14:00 IST, IEC shows enhancement after sunset during high solar activity. This phenomenon is generally called post sunset secondary maximum (PSSM) and has been studied at equatorial and low-latitudes by Tyagi et al. (1982), Lakha Singh (1990a) and Gupta and Singh (2000).

Sometimes intense and rapid fluctuations in the Faraday rotation angle, called Faraday polarization fluctuations (FPFs), have also been observed during post sunset hours accompanying the amplitude scintillations during high solar activity. Many scientists such as Klobuchar and Aarons (1980), Maitra et al. (1984), Das Gupta et al. (1982), Das et al. (1988), Vijayakumar et al. (1988), and Rama Rao et al. (1993) have reported intense and fast fluctuations of the Faraday rotation angles in association with strong and fast amplitude scintillations, respectively. Although the phenomena of PSSM, amplitude scintillations and FPFs have been studied in one form or another, a unified picture has yet to emerge. From the preliminary examination of Delhi data, Lakha Singh et al. (1990a) reported that FPFs are observed only on those days when both PSSM and amplitude scintillations are present. Dabas et al. (1998) also reported that the intensity of the $4 \mathrm{GHz}$ scintillations at Sikandrabad (a station near Delhi) is strongly dependent on the occurrence of PSSM in IEC, and their day-to-day occurrence is strongly dependent on the evening hours' $h$ ' $F$ and its rate of increase over the magnetic equator. In this paper, an attempt is made to further extend the study of the simultaneous occurrence of these parameters and to critically examine the dependence of FPFs on PSSM and the occurrence of amplitude scintillations. The conditions to produce their simultaneous occurrence at Delhi are also discussed in relation to daytime electrojet strength and the evening hour h'F variations over the magnetic equator.

\section{Data and method of analysis}

Faraday rotation and amplitude scintillation data for the period May 1978-December 1980, obtained from VHF telemetry transmissions from geostationary satellites, such as Symphonie-II (May 1978-April 1979) at $137 \mathrm{MHz}$ and 


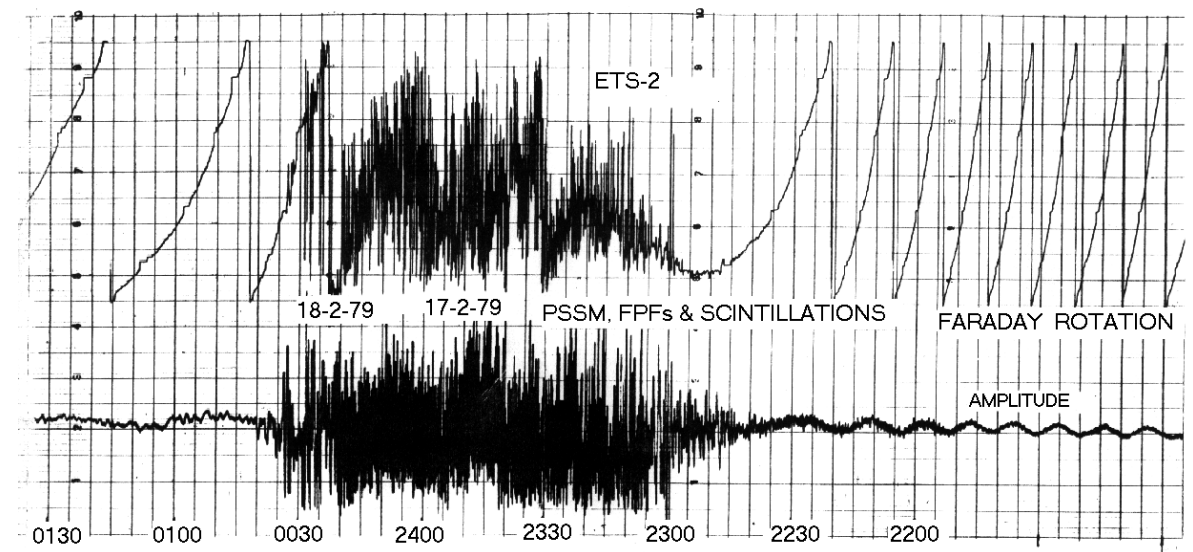

Fig. 1. Faraday rotation and amplitude records showing PSSM, scintillations and FPFs altogether.
ETS-II (May 1979-December 1980) at $136 \mathrm{MHz}$ recorded at National Physical Laboratory (NPL), Delhi, have been utilized for the present study. No data could be recorded during the months of March 1980, and September 1979 and 1980 due to satellite ecliptic conditions. The days for which both FR and amplitude data are available at NPL have been considered for the present study. The data have been surveyed for the presence of PSSM and if present, the time of occurrence of its peak, duration and peak amplitude has been noted, along with the time of occurrences of the amplitude scintillation and FPFs. To examine the relationship between the occurrence of PSSM, FPFs and scintillations with daytime electrojet strength variation, the electrojet strength is calculated using the scheme suggested by Chandra and Rastogi (1974) and the detailed procedure is described by Gupta and Singh (2000) and the references therein. In addition, ionosonde h'F data from an equatorial station of Kodaikanal (dip $3.5^{\circ}$ ) has also been utilized.

\section{Observations and results}

The IEC shows diurnal variation with a maximum around 14:00 IST and minimum around 05:00 IST. However, during the equinoctial and winter months of high solar activity at Delhi after reaching its daytime maximum in the early afternoon, instead of its continuous rapid decay, the IEC sustains for a few hours and often starts to show secondary enhancements after the ionospheric sunset (from 18:00 IST onwards), reaching another maximum centered around 20:00 IST. This phase of secondary maximum lasts for 1-3 $\mathrm{h}$ (approximately).

In a number of cases, it is observed that along with or a few minutes after the onset of PSSM, intense and fast fluctuations in the Faraday rotation angle (FPFs) also take place. In viewing the amplitude record, it is found that during this time, intense and fast scintillations also occur (Lakha Singh et al., 1990a). During the presence of FPFs, it is almost impossible to count the Faraday rotations and also to find the fading rate of scintillations in that time interval. After the FPFs are over, the IEC starts to decrease again and follows the course of normal diurnal variation. In addition, from the analysis of the ATS-6 satellite IEC data, it was noted that no PSSM or FPFs are observed during October 1975-July 1976, which is a low solar activity period.

At Delhi, during May 1978-December 1980, a total of 535 days of simultaneous FR and amplitude records are available for the present study. Out of these 535 days of simultaneous data, PSSM occurs on 205 days, whereas PSSM and FPFs, together with amplitude scintillations, only occur on 64 days. No FPFs are observed on the days when PSSM was absent. Figure 1 contains a typical Faraday rotation and amplitude records showing the simultaneous occurrences of PSSM, scintillations and FPFs. This record corresponds to the night of 17/18 February 1979. On this day, after the diurnal maximum, FR decreases down to 22:45 and starts rising again from 22:45 IST (showing the presence of PSSM). During this time, at about 22:45 IST, the scintillations set in. At 22:55 IST, FPFs appear and suddenly, the FR is unidentifiable, making it almost impossible to count the Faraday fades up to 00:30 IST. The onset of the amplitude scintillation is seen 10 min earlier than the onset of the FPFs and continues up to 00:40 IST and stops abruptly at 00:40 IST. Where the observations correspond to a larger duration (30 min or more) of scintillations, the response of FPFs is very good. Observations also show that scintillations may occur even during post midnight hours, but that FPFs occur only during premidnight hours. All the FPFs events are observed when both PSSM and scintillations are duly present, but the opposite is not true.

Figure 2 shows separately the percentage occurrence of PSSM, scintillations and FPFs in each month from May 1978 to December 1980. It shows that the occurrence of PSSM is maximum followed by scintillations and FPFs in winter and in the equinox, respectively. It also shows that there are no PSSM and FPFs in summer, whereas nighttime scintillations are often present during summer. PSSM peaks equally high in winter and in the equinox, whereas FPFs peak in the equinox, followed by winter in high solar activity at Delhi. During low solar activity period (ATS-6 data), no PSSM and FPFs have been observed. The PSSM, scintillations and 
FPFs show (a) seasonal and (b) solar activity dependence, as can be seen from Fig. 2 .

In order to see the general trend of variation, the monthly percentage occurrence of PSSM, scintillations and FPFs have been obtained for January to December (12 months) by combining and averaging the corresponding data of the same month in all three years (1978, 1979 and 1980), plotted as months versus PSSM, scintillations and FPFs in Fig. 3a, $\mathrm{b}$, and $\mathrm{c}$, respectively. Figure $3 \mathrm{a}$ shows that PSSM occurrence has two strong maxima in autumn and spring. These equinoctial maxima are separated by a broad summer minimum. Figure $3 b$ shows that scintillation occurrence is maximum in the summer months and decreases during winter and the equinox months (obvious on either side of the summer occurrence). This is almost the reverse of the situation in the case of the PSSM occurrence. From Fig. 3c it is found that the occurrence of FPFs is almost the same in the equinox and winter months, but they are totally absent in summer at Delhi. This is comparable with the PSSM seasonal occurrence.

It is pointed out here that the characteristics of scintillations observed at Delhi during summer are different than that observed during the winter and equinoctial periods. During summer, scintillations start just after local sunset and their onset is gradual and can continue even throughout the night. Scintillations observed during equinoctial and winter periods are characterized by their sudden onset and abrupt death, and are mostly observed in patches one or two hours after local sunset and are generally confined up to the local midnight hours, especially during peak solar activity periods (Dabas et al., 1991; Gupta et al., 1993). At the Delhi latitude, winter and equinoctial scintillations, especially during the high sunspot years, are generated by equatorial plasma bubbles associated with irregularities, whereas summer scintillations, which are not dependent on solar activity conditions, are caused by irregularities generated locally (Dabas et al., 1991). Based on the results of Figs. 2 and 3, it is noted that the occurrence of PSSM and FPFs are minimum during summer and have no association with summer scintillations, but are strongly dependent on the solar activity conditions. The occurrence of PSSM and FPFs during winter and equinoctial periods are maximum during peak solar activity period. It is also noted that the occurrence of FPFs are always associated with simultaneous occurrences of PSSM and scintillations. FPFs are observed primarily during the period of intense scintillation activity.

\section{Relationship between daytime electrojet strength and the occurrence of PSSM, scintillations and FPFs}

In recent years, several authors have tried to see the association between the strength of the daytime equatorial ionization anomaly and the occurrence of equatorial and lowlatitude scintillation. This is basically to gain prediction capabilities of the occurrence of scintillations in the equatorial and low-latitude region. The strength of the equatorial ionization anomaly depends upon the electrojet strength. It is,

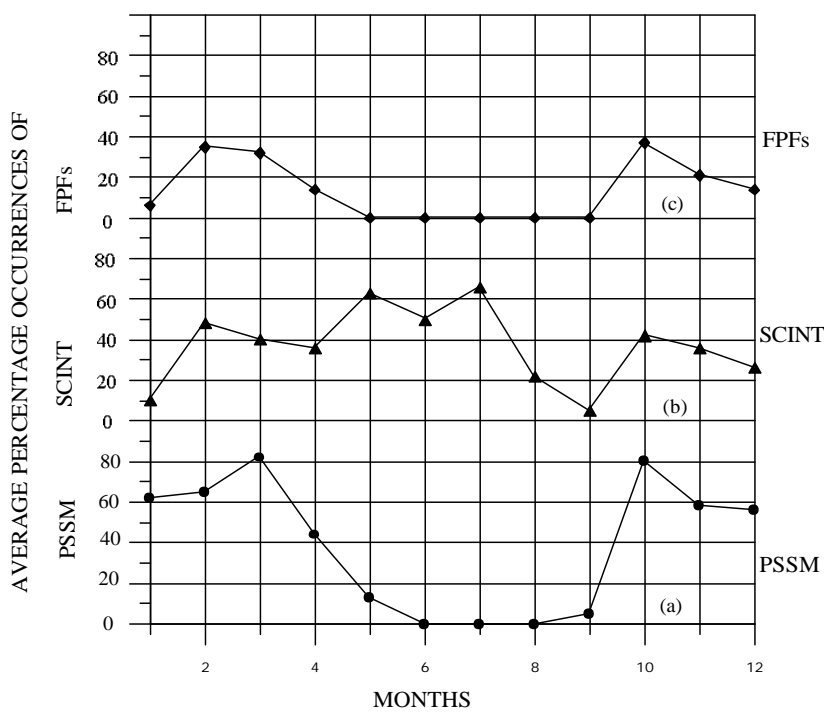

Fig. 2. Diagram showing monthly occurrence of PSSM, scintillations and FPFs.

therefore more suitable to use electrojet strength for this purpose. In addition, it is well established that during equinox periods of high sunspot years, scintillations at Delhi or even beyond are caused by equatorial plasma bubbles and associated irregularities (Somayajulu el al., 1984; Dabas et al., 1991) and their occurrences are strongly dependent on the evening hours h'F variation over the magnetic equator. To examine the influence of the daytime electrojet strength (EEj) on the occurrence of PSSM, scintillations and FPFs, we have used here only October 1979 data, where the occurrence frequency of PSSM, scintillations and FPFs was observed to be maximum (see Figs. 2 and 3). Figure 4 shows the plots of the daily values of $A_{p}$, EEj at 11:00 IST, along with the occurrence of PSSM, scintillations and FPFs observed at Delhi. In addition, in order to examine their association with the evening hours h'F variations near the magnetic equator, h'F (19:00 IST) values from an equatorial station, Kodaikanal, are also plotted in Fig. 4, where it is noted that the simultaneous occurrence of PSSM, scintillations and FPFs takes place when EEj was $100 \mathrm{nT}$ or more, whereas when EEj was less than 50, none of them were observed. It is interesting to note that $\mathrm{EEj}$ and h'F variations also follow the same trend, i.e. when EEj is more, h'F is also more. It is also noted that PSSM, scintillations and FPFs are simultaneously observed only when h'F was around $500 \mathrm{~km}$. The magnetic disturbance seems to decrease both the EEj and the h'F. In addition, as can be seen from Fig. 4, that both FPFs and scintillations are observed only when PSSM is present.

\section{Discussion}

The present results discussed above indicate that as far as the simultaneous occurrence of PSSM, scintillations and FPFs is concerned, it is found that FPFs occur only on those days 


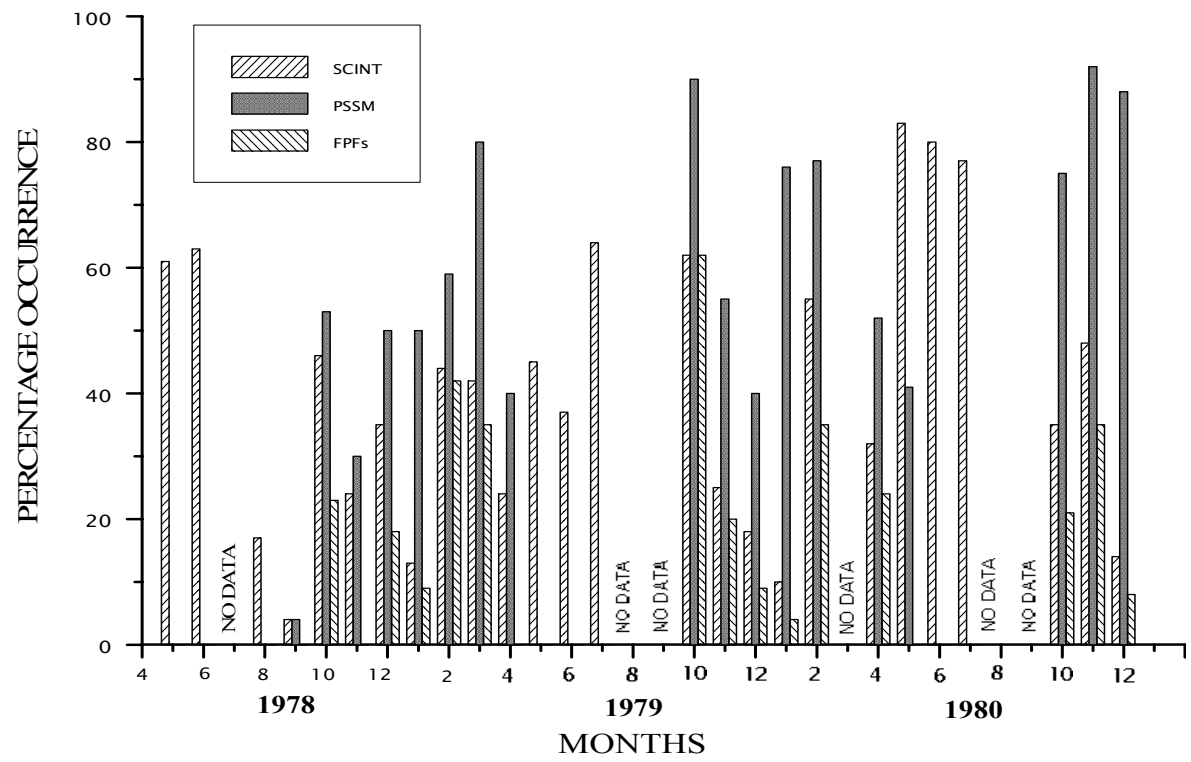

Fig. 3. Diagram showing monthly average occurrence (of 1978, 1979 and 1980) of (a) PSSM, (b) scintillations and (c) FPFs. when both PSSM and amplitude scintillations are present. Although the scintillations are also found to occur in the absence of PSSM, FPFs are found to occur only on PSSM days. In addition, on most of the occasions, FPFs and scintillations occur simultaneously or with a gap of about $10 \mathrm{~min}$, with the former occurring later and dying in the reverse order.

At low-latitudes, Rao et al. (1963), Sastri (1982) and Garg et al. (1983) reported that the post sunset enhancements in the IEC during high solar activity are related to the upward movements of the layer and the strengthening of the equatorial fountain, thus underlying the role of $\boldsymbol{E} \times \boldsymbol{B}$ drifts at low-latitudes. Basically, the post sunset increase in the IEC has its bearing on the electric fields, which play an important role in shaping the electron density distributions in the equatorial region. They drive the electrical fountain that produces the equatorial trough or Appleton anomaly, which is a striking feature of the low-latitude ionosphere. Normally, the daytime eastward electric field changes to a westward direction, after sunset, but during high solar activity, the eastward electric field in the F-region of the equatorial ionosphere often shows a significant and fairly sharp increase just before it reverses to its nighttime westward direction. At post sunset, the E-region electron density drastically drops. During this time, thermospheric winds are strong and the F-region electron density is still quite high, leading to a strong F-region dynamo, which produces polarization fields that are perpendicular to the geomagnetic field and currents along the field lines in the equatorial region. The driving force for the polarization field, which generates the vertical drift or zonal electric field, is the zonal neutral wind. Goel et al. (1990) have shown that the polarization field is dependent upon the Eregion density, so its effect will be maximum when both the conjugate points in the E-region correspond to the F-region at the equator sunset. Thus, during equinoxes, with the sunset in the E-region being simultaneous, polarization fields that developed in the equatorial F-region are maximum, showing a prereversal enhancement to be maximum and consequently, the post sunset IEC value. This explains the maximum occurrence of the IEC during equinox.

Klobuchar and Aarons (1980) and Maitra et al. (1984) suggested that FPFs at $136 \mathrm{MHz}$ are due to the depolarization effect caused by power-law type density irregularities, with scale size in the range of 50-200 $\mathrm{m}$ in a background of high ionization density. Lakha Singh et al. (1990b) compared the occurrence of FPFs and scintillations at Delhi with the corresponding data from a low-latitude station of Hyderabad and noted that the occurrence of both FPFs and the scintillation at Delhi is conditional to their prior occurrence at the lower latitude station, Hyderabad. Dabas et al. (1998) also reported that at Sikandrabad (a station near Delhi), the intensity of $4 \mathrm{GHz}$ scintillations is strongly dependent on the occurrence of PSSM in the IEC, and their occurrence during equinoxial periods of high sunspot years is strongly dependent on the evening hours' h' $F$ and its rate of increase over the magnetic equator.

The current results of the simultaneous occurrences of FPFs and scintillations in the presence of an enhanced background ionization are in line with the results discussed above. Their characteristics and occurrence patterns suggest that these are caused by an equatorial plasma bubble and the associated irregularities generated initially over the magnetic equator and subsequently mapped along magnetic field lines to low-latitudes, especially during the equinoctial period of high sunspot years. In addition, it is interesting to note that the day-to-day variation in the occurrences of FPFs and scintillations at Delhi is well associated with the daily variations in the daytime electrojet strength and evening hours of h'F values over the magnetic equator. These are more likely to occur when EEj is $100 \mathrm{NT}$ or more, and h'F reaches $500 \mathrm{~km}$ or more. These two parameters are very important and can 


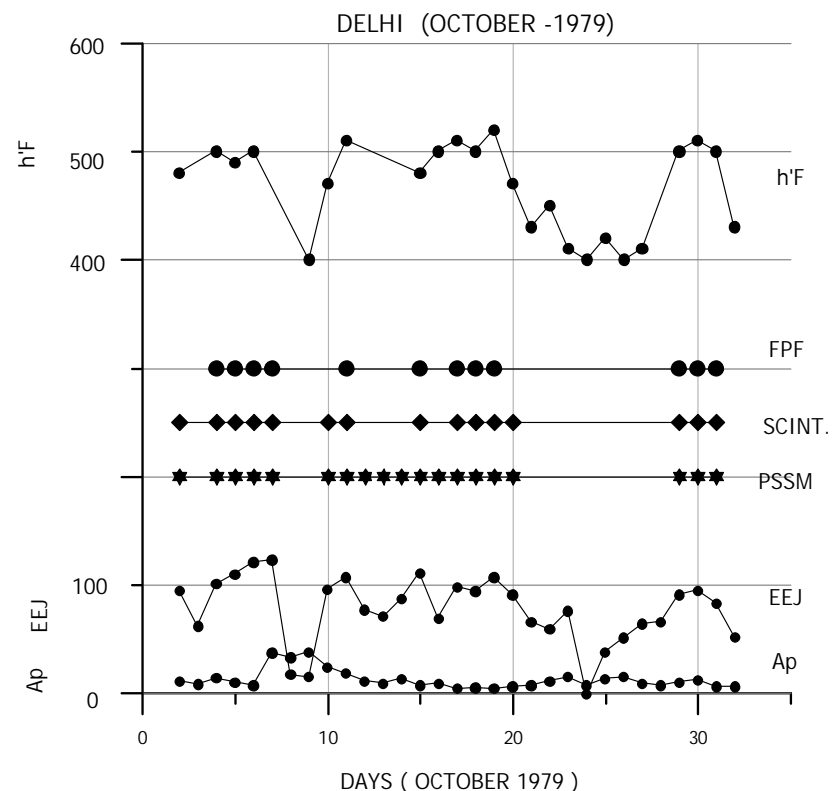

Fig. 4. Diagram showing the daily values of $A_{p}$, electrojet strength (EEj) and h'F at an equatorial station, Kodaikanal, for the month of October 1979. In addition, (for comparison) the occurrences of PSSM, scintillations and FPFs are shown on a daily basis.

be used for predicting the FPFs and scintillation occurrences at low-latitudes.

At this point, it is also important to view the relationship between the scintillations and FPFs. At the outset, these are caused by irregularities with different scale sizes. When strong VHF amplitude scintillations are considered alone, the order of the scale size involved is a $\mathrm{km}$, while FPFs are caused by irregularities of scale sizes of the order of 50$200 \mathrm{~m}$. The equatorial irregularities during the post sunset development phase follow a power law density spectrum. In order to cause VHF depolarization, the irregularities in the scale size range of 50-200 m should have sufficient scattering power; in other words, the overall strength of the irregularities should be very intense. It is also noted that the overall strength of the irregularities is high when the background ionization is more, i.e. in the presence of PSSM. The fact that the temporal extent of scintillations is always invariably larger than that of the FPFs shows that the irregularities of smaller scale sizes evolve and decay in a much shorter time (an hour or two), compared to the kilometer size irregularities that may continue to exist even after midnight hours, thus giving rise to weaker scintillations.

\section{Summary}

The results obtained from FR and the amplitude monitored at Delhi during high solar activity shows that:

1. The occurrence of FPFs at Delhi is conditional to the simultaneous occurrence of both PSSM and scintillations.
2. The occurrence of FPFs is a pre-midnight feature, whereas scintillations may occur throughout the night. The scintillations associated with FPFs are characterized by a deep fading (SI $>60 \%$ ) with a fast fading rate (about 10-15 fades/min).

3. The onset of scintillations (associated with FPFs) is often abrupt, with explosive growth reaching fluctuation levels of $10-15 \mathrm{~dB}$ within a few minutes.

4. The comparison of Delhi and Hyderabad results show that the occurrence of FPFs and scintillations at Delhi is conditional to their prior occurrence at Hyderabad (a low-latitude station within the equatorial anomaly zone), especially during equinoctial periods of high sunspot years.

5. The occurrence of PSSM and FPFs takes place only in the winter and equinox seasons of high solar activity and they are totally absent during summer at Delhi.

6. The occurrence of FPFs and scintillations at Delhi are strongly dependent on the daytime electrojet strength variations and are observed only on those days when $\mathrm{EEj}$ is $100 \mathrm{nT}$ or more.

7. Evening hours h'F values are well associated with daytime EEj strength at an equatorial station.

From the present study, we find that FPFs are observed when irregularities are intense and occur over a large spatial extent in the vertical direction. During the developmental phase in the post sunset and pre-midnight hours, the equatorial irregularities in the form of plasma bubbles are very strong and occupy several hundred $\mathrm{km}$ of the topside ionosphere. Irregularities with scale sizes extending over hundreds of $\mathrm{km}$ to $\mathrm{cm}$ coexist in this phase. The ambient plasma density at locations near the Appleton anomaly crests is higher than those at the equatorial stations by a factor of 2-4 during the high solar activity period. This, together with $\mathrm{km}$ size irregularities and very intense irregularities of the size of 50-200 m, creates a favourable atmosphere for the production of PSSM, scintillations and FPFs at Delhi.

Acknowledgements. Topical Editor M. Lester thanks R. Leitinger and another referee for their help in evaluating this paper.

\section{References}

Chandra, H. and Rastogi, R. G.: Geomagnetic storm effects on ionospheric drifts and the equatorial $\mathrm{E}_{s}$ over the magnetic equator, Ind. J. Radio Space Phys., 3, 332-336, 1974.

Dabas, R. S., Banerjee, P. K., Bhattacharya, S., Reddy, B. M., and Singh, J.: Gigahertz scintillation observations at $22 \mathrm{~N}$ magnetic latitude in the Indian zone, Radio Sci., 26, 759-771, 1991.

Dabas, R. S., Lakshmi, D. R., and Reddy, B. M.: Day-to-day variability in the occurrence of equatorial and low latitude scintillations in the Indian zone, Radio Sci., 33, 89-96, 1998. 
Das, S. K, Das Gupta, A., Maitra, A., and Chakraborty, S. K.: Post sunset ambient ionization and ionospheric scintillations in the equatorial region under solar activity maximum and minimum conditions, Proc. International Beacon Satellite Symposium on Investigation of the ionosphere by means of Beacon satellite measurement, Beijing, China, 50-57, 1988.

Das Gupta, A., Lee, M. C., and Klobuchar, J. A.: VHF Faraday polarization fluctuations and strong L-band amplitude scintillations near Appleton anomaly crests, Nature 298, 354-356, 1982.

Garg, S. C., Somayajulu, Y. V., Lakha Singh, and Tyagi, T. R.: Evidence of the development and decay of a post sunset equatorial anomaly at low-latitudes, Proc. International Symposium on "Beacon Satellite Studies of the Earth's environment", New Delhi, 359-373, 1983.

Goel, M. K., Singh, S. S., and Rao, B. C. N.: Post sunset rise of Flayer height in the equatorial region and its relation to the F-layer dynamo polarization fields, J. Geophys. Res., 95, 6237-6246, 1990.

Gupta, J. K., Lakha Singh, and Tyagi, T. R.: Study of scintillations observed at Delhi during high solar activity period, Ind. J. Radio Space Phys., 22, 193-196, 1993.

Gupta, J. K. and Lakha Singh: Long term ionospheric electron content variations over Delhi, Ann. Geophysicae, 18, 12, 16351644, 2000.

Klobuchar, J. A. and Aarons, J.: Studies of equatorial irregularity patches using SIRIO VHF transmissions, Alta Freq., 49, 345349, 1980.

Lakha Singh, Gupta, J. K., Vijayakumar, P. N., and Tyagi, T. R.: Morphological study of post sunset secondary maximum in ionospheric electron content and its correlation with Faraday polarization fluctuations and VHF scintillations at Delhi, Proc. International Beacon Satellite Symposium, Tucuman, Argentina, 5154, 1990a.
Lakha Singh, Gupta, J. K., and Tyagi, T. R.: A comparative study of post sunset secondary maximum in ionospheric electron content and Faraday polarization fluctuations during high solar activity period, paper presented at International Symposium on "Optical and Radio remote sensing of the atmospheric environment", National Physical Laboratory, New Delhi, 24-26 October, 195-196, 1990b.

Maitra, A., Das, S. K., and Das Gupta, A.: Observations of fast Faraday polarization fluctuations and VHF amplitude scintillations at VHF and UHF near the equatorial anomaly crest in the Indian sector, Radio Sci., 19, 725-730, 1984.

Rama Rao, P. V. S., Ramana Rao, B. V., and Prasad, D. S. V. V. D.: Study of ionospheric electron content depletions and their association with VHF scintillations, Ind. J. Radio Space Phys., 22, 259-266, 1993.

Rao, B. C. N.: The post sunset rise of $f o \mathrm{~F} 2$ in the transition region and its dependence on the post sunset rise of h'F in the equatorial region, J. Geophys. Res. 68, 2551-2557, 1963.

Sastri, J. H.: Post sunset behaviour of the equatorial anomaly in the Indian sector, Ind. J. Radio Space Phys., 11, 33-37, 1982.

Somayajulu, Y. V., Garg, S. C., Dabas, R. S., Lakha Singh, Tyagi, T. R., Lokanadham, B., Ramakrishan, S., and Navneeth, G.: Multistation study of nighttime scintillations in low-latitudes: Evidence of control by equatorial F-region irregularities, Radio Sci., 19, 707-718, 1984.

Tyagi, T. R., Yeh, K. C., Tauriainen, A., and Soicher, H.: The electron content and its variations at Natal, Brazil, J. Geophys. Res., 87, 2525-2532, 1982.

Vijayakumar, P. N., Tyagi, T. R., and Gupta, J. K.: Salient features of amplitude scintillations and Faraday polarization fluctuations at VHF observed at Delhi, Ind. J. Radio Space Phys., 17, 58-62, 1988. 